\title{
Temporal and spatial factors in gait perception that influence gender recognition
}

\author{
CATHARINE D. BARCLAY, JAMES E. CUTTING, and LYNN T. KOZLOWSKI \\ Wesleyan University, Middletown, Connecticut 06457
}

\begin{abstract}
Several temporal and spatial factors affect gender recognition of a walker when portrayed, without familiarity cues, as a dynamic point-light display. We demonstrate that, among temporal parameters, the duration of the dynamic stimulus. must be longer than $1.6 \mathrm{sec}$, but that $2.7 \mathrm{sec}$ is fully adequate. Given the speed of our walkers, the recognition threshold appears to be roughly two step cycles. In addition, presentation rate of the stimulus must be near to normal, perhaps because nonnormal rates alter apparent gravity and obscure the normal relationship between output and conservation of energy. We demonstrate that, among spatial factors, the discreteness of the joint information must be maintained for accurate recognition. We go on to argue that it is the information about the shoulder and the hip of a walker that is of primary importance. Finally, inversion of the stimulus display produces the unexpected effect of reversing the apparent sex of most walkers. That is, when presented upside down, male walkers appear female and female walkers appear male.
\end{abstract}

If one removes familiarity cues from a dynamic display, nothing is left for the viewer to perceive except formless relations projected over time. Yet these formless relations can yield rich and vivid percepts. This fact proves embarrassing for any view of form perception that relies exclusively on superficial features such as lines and angles. The present studies explore how formless relations that afford gender recognition in human walkers survive temporal and spatial distortion. Our goal is that through this type of study we can learn more about the nature of those relations and how they are perceived.

For nearly a century researchers have attached points of light that glow in the dark to the limbs of walkers for the purposes of studying gait (see Bernstein, 1967). In fact, through this technique and others, human gait may be the most studied of all biological movement. Nevertheless, nearly all of this work has focused on how the movement is produced. Until the work of Johansson (1973, 1975, 1976), few have studied how that movement is perceived.

Using a point-light technique developed by Johansson, we have begun to study various aspects of gait recognition. Previously, we had found that individuals can recognize themselves and others without familiarity cues from such a dynamic display (Cutting \& Kozlowski, 1977). Next, we (Kozlowski \& Cutting, 1977) determined that viewers could iden-

This paper is based on the Master's thesis of the first author. This research was supported by a small research grant from Wesleyan University to each of the authors. We thank Robert $J$. White for his continued help, and Carol Fowler, Dennis Proffitt, and David Stier for instructive comments. Reprint requests should be sent to J. Cutting or L. Kozlowski, Department of Psychology, Wesleyan University, Middletown, Connecticut 06457. tify the gender of a walker from that display, but not from static tokens taken out of the dynamic sequences. Variations in walking speed and in armswing generally detracted from sex recognition. However, such recognition was possible from minimal cues, such as those placed on the ankles alone. The studies presented here are a continuation of that research. We pursue two temporal cues influencing gender recognition of a walker, the duration of stimulus exposure and its presentation rate, and two spatial cues, the discreteness of the point sources of information and the orientation with respect to gravity.

\section{GENERAL METHOD}

The techniques used here were the same as those employed by Johansson (1973), modified by Cutting and Kozlowski (1977) and Kozlowski and Cutting (1977). The equipment included glassbead retroreflectant tape wound around walkers' joints, videotape recording and monitoring devices, and high-intensity lights mounted near the lens of the television camera and focused on the designated walking area.

For purposes of generality, we used a larger sample of walkers than did Kozlowski and Cutting (1977): 14 Wesleyan University undergraduate students, 7 males and 7 females. Each walker appeared to have a normal gait, and all were approximately the same height and weight. Respective ranges for males were 1.68 to $1.75 \mathrm{~m}$ and 54.1 to $65.9 \mathrm{~kg}$; those for females were 1.70 to $1.75 \mathrm{~m}$ and 52.3 to $64.2 \mathrm{~kg}$. Since males are typically larger than females, their comparability to females in height and weight in these studies provides a strong test for the hypothesis that gender recognition can be accomplished through an analysis of formless dynamic relations.

All walkers wore dark turtleneck shirts and dark pants during the recording sessions. Males and females were videotaped in separate evening sessions, 2 weeks apart. The male walkers were recorded by the three authors and two male assistants. As a precaution against possible over self-consciousness among female 
walkers, no males participated in the second recording session; instead, the first author and two female assistants ran the session. ${ }^{1}$

Five-centimeter-wide Scotchlite reflectant tape was wrapped around the walker's wrists, around his or her arms just above the elbow, around the ankles, and around the legs just above the knee. Strips of tape, $5 \times 15 \mathrm{~cm}$, were attached to the waist and to the shoulders as epaulets, half on the shoulder and half on the upper arm. No tape was placed on the head. Each individual walked at normal speed for several minutes until the recording team was satisfied that he or she was not "performing" in front of the camera. Side views of walkers were recorded on a source tape as each passed left to right in a straight line before the camera, approximately $6 \mathrm{~m}$ from the lens. Two separate tokens were used for each walker. Individual walkers were on camera for 7 strides $( \pm 1 / 2$ stride $)$ and a mean of $4.4 \mathrm{sec}(\sigma=.37)$ for each traverse across the viewing field. The gait of each individual was recorded separately, and the television camera remained in a fixed position without panning to follow the walker's steps. During recording, video contrast was turned to maximum and brightness to near minimum. Equipment used in recording sessions included a GBC Viewfinder television camera (Model VF-302), a helicalscan Sony-Matic solid-state videorecorder (Model AV-3650), a 23-in. Setchell-Carlson television monitor (Model 3ER1200), and two Berkey Colortran Multi-10 lights (Model 100-301).

Test sequences were produced by recording from the source tape onto a second videotape. Equipment used in production of the test tape included two videorecorders, the Sony and a Javelin (Model X-400), two 23-in. monitors attached to both the recorder on which the source tape was played and to the one on which the test sequence was recorded, and a Telemation television camera (Model TMC-2100V) focused on one monitor at close range and connected to the test-sequence recorder. 'A test trial was first located on the source tape with the aid of an audio channel dubbed onto it after the recording session. Then that trial was played on the source tape recorder and recorded onto the test tape. The next trial was then located on the source tape and the procedure repeated until all trials were recorded. To guarantee that both recorders reached running speed, ensuring no flopover of image within a trial, an interval of about $15 \mathrm{sec}$ was placed between trials. Barrel distortion and other image degradation resulting from the rerecording process were minimal. Each test trial was announced by number, dubbed onto the audio track.

Male and female Wesleyan University undergraduate students served as viewers in all five experiments. In general, they were run in groups of 4 to 10 , and no viewer served in more than one experiment. In the experimental session a 9-in. Sony solidstate television monitor (Model PJV-51RU) was used. After viewing each trial, the observers wrote down $M$ or $F$ to indicate the sex of the walker, and then indicated confidence in their judgments on a 5-point unipolar scale, with 5 representing high confidence and 1 a guess. Visual angle, measured vertically, ranged from about $.5^{\circ}$ to $4^{\circ}$ across all experimental groups. Just as no feedback was given in the Johansson and the Cutting and Kozlowski studies, none was given to viewers in any of the experiments described here.

In all experiments but the last, a standard condition is compared against one or more conditions in which one stimulus dimension is manipulated. The standard condition always (a) consists of full screen presentation $(4.4 \mathrm{sec})$ of the walkers, (b) played at a tape speed of $20 \mathrm{~cm} / \mathrm{sec}$, (c) with all joints represented as single lights, (d) on an upright monitor. In other conditions, one factor is altered per experiment.

\section{EXPERIMENT 1 VARIATION IN EXPOSURE DURATION}

Johansson (1976) reported that subjects were able to organize the moving point-light configuration into a "gestalt" of a walking human figure within
$200 \mathrm{msec}$. If such brief exposures are sufficient for the perception of a person in motion, perhaps they are also sufficient for accurate gender recognition.

\section{Method}

Four different randomized test sequences were constructed with 28 trials each. Exposure durations were 4.4, 1.6, .8, and .4 sec. Each walker was represented by two different tokens in each sequence. Eight practice trials preceded the 4.4-sec, or standard, test; none preceded the other three. The standard sequence presented walkers as they moved across the entire monitor screen. All three sequences of briefer exposure were created by placing a Pronto Press tachistoscopic shutter over the lens of the television camera when the test sequences were recorded. Its aperture was opened to maximum, and its timer set to the appropriate shutter speeds. Specific durations $(1.6, .8$, and $.4 \mathrm{sec})$ were determined later by electronic measurement. Just as Johansson (1976) made no attempt to align stimulus onset or offset with particular points in the gait cycle, we too made no such attempt for the brief exposure sequences. No ready signal occurred between trials; only the near-invariant thythm of the sequence cued the viewers.

As a group class project, 57 participants $(26$ males and 31 females) viewed the four test sequences in the order of decreasing exposure duration.

\section{Results and Discussion}

The standard sequence confirmed results found with the smaller stimulus sample of Kozlowski and Cutting (1977). Overall, viewers correctly identified the gender of walkers on $66 \%$ of all trials, $t(56)=$ $11.0, \mathrm{p}<.001$, two-tailed. ${ }^{2}$ Chance performance is, of course, $50 \%$. Viewer performance decreased sig. nificantly with exposure, $\mathrm{F}(3,168)=61.0, \mathrm{p}<.001$. Correct identification at an exposure of $1.6 \mathrm{sec}$ was only $52 \%, \mathrm{t}(56)=1.11$, n.s., and at briefer exposures of .8 and $.4 \mathrm{sec}$ it was reduced to below chance. At $.8 \mathrm{sec}$, accuracy was $46 \%, \mathrm{t}(56)=-3.04$, $\mathrm{p}<.01$, and at $.4 \mathrm{sec}$, it was only $45 \%, \mathrm{t}(56)=$ $-3.49, p<.001$. Both of these anomalous effects, however, are attributable to systematic misidentification of two female walkers. When they are removed from statistical consideration, performance at the two brief exposures does not differ statistically from chance. It is not clear, however, what provoked misidentification of these two walkers.

Comparison across the four exposure durations reveal significant differences in viewers' ability to perform the task. Performance for the standard $(66 \%)$ and 1.6-sec $(52 \%)$ durations differed reliably, $\mathrm{t}(56)=9.0, \mathrm{p}<.001$, as did performance at $1.6 \mathrm{sec}$ from the two briefer exposures, $\operatorname{ts}(56)=3.29$ and $3.50, p<.01$. Performance at the two briefer exposures did not differ statistically.

Confidence judgments reflected viewer performance, just as they did in the studies of Kozlowski and Cutting. Mean confidence scores at the four exposures were $2.13,1.78,1.39$, and 1.15 , respectively, $\mathrm{F}(3,165)=71, \mathrm{p}<.001$. The standard sequence, however, was the only one for which viewers' confidence scores for correct (2.31) and incorrect (1.89) trials differed, $F(1,55)=12.2, \mathrm{p}<.001$. 
Of secondary importance are the interactions with gender. First, there was an interaction between exposure duration and gender of the walker, $F(3,168)$ $=3.31, \mathrm{p}<.05$. That is, at a duration of $4.4 \mathrm{sec}$, males proved somewhat less identifiable $(64 \%)$ than females $(68 \%)$. This effect is almost certainly due to response bias, and no such difference occurred at other durations or in other experiments reported here. Second, in the standard condition, female viewers performed better $(71 \%)$ than did males $(61 \%), t(56)=6.0, p<.001$. In general, this effect occurs in all standard and many experimental conditions throughout this paper, although always to a lesser degree. It may be that women try harder in this type of experiment. Nonetheless, since this type of difference is not a major focus of these studies, it will not be discussed again.

In summary, viewers are able to identify the gender of walkers from 4.4-sec dynamic displays. At briefer exposures, however, identifiability is reduced to near chance. Thus, while Johansson demonstrated that exposures as brief as $.2 \mathrm{sec}$ are sufficient for identification of a moving human figure from a point-light display, it is apparent that gender recognition requires considerably longer. To understand why more than $1.6 \mathrm{sec}$ might be necessary, it is useful to consider the biomechanics of gait, particularly the step cycle.

The step cycle. Carlsöö (1972) and others (Farber, 1969, pp. 103-113; Napier, 1967; Steindler, 1935) provide an informative description of human gait. It is a modification of the Philippson step cycle used in the analysis of animal gait (see Grillner, 1975). At the beginning of each step, the walker stands on one leg while swinging the other leg forward. This is called the single-support phase. Next, he steps on that leg and, for a brief time, is supported by both legs as weight is transferred from back leg to front. This is called the double-support phase. This same pattern is then repeated on the other side-single-support followed by the double-support phase. The step cycle is ended when all body parts are in the same position as at the beginning of the cycle. Thus, the cycle takes two full steps to complete. If sex recognition is related to the step cycle, more information is needed about the walkers in this study, as well as those of Kozlowski and Cutting (1977).

Consider two parallels from the two samples. The first concerns walking speed. Kozlowski and Cutting's 6 walkers averaged $101.3 \mathrm{paces} / \mathrm{min}(\sigma=7.9)$, and the 14 walkers in the present study averaged 100.1 paces $/ \min (\sigma=9.1)$, with no reliable difference between groups. These walking speeds are faster than the normal rates reported by Beckett and Chang (1969) and Carlsöö (1972), but they are consistent with the notion proposed by Bornstein and Bornstein (1976)-lifestyle affects walking speed. It is not unlikely that Wesleyan undergraduates live more stress- ful lives than do the peasants of, say, Corsica and Crete, and that this contributes to increase walking speed. The second parallel between the samples is seen in the identifiability of walkers in both studies: Overall, Kozlowski and Cutting found 63.2\% $(\sigma=$ 16.8) correct identification and we found $65.1 \%$ $(\sigma=8.2)$, again a nonsignificant difference.

Given a walking speed of about 100 paces $/ \mathrm{min}$, each stride takes approximately $.6 \mathrm{sec}$, and a complete step cycle $1.2 \mathrm{sec}$. In the present study, viewers could identify the sex of walkers within $4.4 \mathrm{sec}$. This translates into roughly 3.7 step cycles. However, they were not able to recognize the gender of walkers within $1.6 \mathrm{sec}$, or roughly 1.3 step cycles. The viewers of Kozlowski and Cutting needed only $2.7 \mathrm{sec}$, or 2.3 step cycles, to make accurate judgments. Since our viewers performed no differently at $4.4 \mathrm{sec}$ than did those of Kozlowski and Cutting at $2.7 \mathrm{sec}$, all information needed for gender recognition would appear to be present within 2.3 step cycles. Thus, more than 1.3 but less than 2.3 cycles would appear necessary, suggesting a threshold of about 2 step cycles.

When viewers perform this type of task, perhaps they use the first step cycle to calibrate the domain of the individual walker's movements and then use the second to confirm that estimate. If the step cycle is a proper unit of analysis for judging gender, then some aspect of the movement within a cycle may be different for males than for females. This notion will be pursued in the general discussion.

The next experiment in this series is directed at a second temporal parameter of the stimuluspresentation rate.

\section{EXPERIMENT 2 \\ NORMAL VS. SLOW PRESENTATION RATE}

Crucial to the perception of any dynamic event is the analysis of its particular rate of change (Jones, 1976). Variation in this rate can alter our perceptual experience. Consider the peculiar effects created by speeding up an event, as seen in the films of the Keystone Cops and Charlie Chaplin, and by slowing it down, as seen in the instant replays of sports television. Altering speed of gait produces particularly odd results. In extreme cases what we see are fast and mechanical robot-like figures, on the one hand, and slow moon-like ones, on the other. The nature of such oddities and their relation to the perception of normal gait provoked this study.

Kozlowski and Cutting (1977, Experiment 4) found that alteration of normal walking speed interfered with sex recognition. Their method required (a) that individuals walk in time to various rates of metronome pulses, and (b) that the rate at which the display was presented to viewers be veridical with that of recording. Thus walking speeds and the re- 
sulting physical movements were always possible, if not portraying the cadence and flow of natural gait. The present study also examines how variation in walking speed affects recognition; however, in this case (a) the natural walking speed of each walker was constant, while (b) stimulus presentation rate was varied. Consequently, these walking speeds and resulting physical movements were not always possible but did, in both cases, depict exactly the same movement.

\section{Method}

A test sequence of 36 trials -8 practice trials followed by a random ordering or two different tokens of each of the 14 walkers - was presented first at a standard and then at a slow speed. The standard trials were identical to those of Experiment 1 . Presentation rate was slowed by adjusting the videorecorder to its slowest possible rate, later determined to be 3.05 times slower than normal. Thus, normal tape speed was $20 \mathrm{~cm} / \mathrm{sec}$ and the slower speed was $6.6 \mathrm{~cm} / \mathrm{sec}$. At standard speed, walkers took $4.4 \mathrm{sec}$ to cross the screen, while at slow speed, they took $13.4 \mathrm{sec}$. Each of seven males and eight females were paid $\$ 1$ to view the two sequences. Unspooling, shuffling, and remounting video tape reels was done between tests to insure that viewers were not aware that they were seeing the same tape twice.

\section{Results and Discussion.}

Viewers were able to identify gender of walkers on $64 \%$ of all standard-speed trials, $\mathrm{t}(14)=4.42, \mathrm{p}<$ .01. Mean confidence score for those trials in which the walkers were correctly identified was 2.2 , while that for incorrect trials was 1.9. As before, this difference, $t(14)=2.7, p<.02$, indicates that viewers can monitor their performance to some degree.

Viewers were unable to identify the sex of walkers when the tape was presented to them at the slower rate. Only $55 \%$ of all items were properly identified, the mean confidence score was 1.9 , while in the case of incorrect trials it was slightly, but not significantly, lower at 1.7. Less confidence in their responses as well as no difference between confidence scores for correct and incorrect trials may reflect viewers' awareness of their inability to perform the task.

The difference in performance at the presentation rates is reliable, $t(14)=1.86, p<.05$, one-tailed, and the difference in confidence scores for correct trials under the two conditions reflects this difference, $t(14)=2.73, p<.01$. One way to understand the performance differences in the two conditions concerns the effect of time change on pendular motion.

Walking as pendular motion. The transformations involved in gait can be described as a series of pendular motions characterized by the displacement of the limbs relative to their respective joints. Consider the movement of the legs: "The pendulum movement is facilitated by the great mobility of the hip joint and occurs largely under the influence of gravity so that very little muscle action is necessary.... The duration of the oscillations, as of any pendulum, depends upon the length and the distribution of the mass" (Steindler, 1935, p. 356). Since gravity supplies much of the force required to overcome the body's inertia in walking and to minimize its expenditure of energy (Beckett \& Chang, 1969; Napier, 1967), an analysis of the relationship between time and gravity seems warranted. All pendulum movement is defined as follows:

$$
\mathrm{T}=2 \pi(\mathrm{L} / \mathrm{g})^{1 / 2},
$$

where $\mathrm{T}$, or time, refers to the duration of a pendular phase, $L$ describes the length of the particular pendulum under consideration as measured from the pivot to the center of mass, and $\mathrm{g}$ is gravity.

The difference in viewers' ability to perform the recognition task at normal and slow presentation rates might be explained in terms of Formula 1 . Under the slow condition of the present study, time was increased by a factor of 3.05 (while in that of Kozlowski and Cutting, 1977, Experiment 4, it was increased by a factor of only 0.18 ). Pendular lengths in the body of a walker, of course, remain basically unchanged. ${ }^{3}$ With such a large change in $\mathrm{T}$, and with no possible completely compensatory change in $L$, gravity must be reduced by a factor of roughly 9.3 . Thus, other factors remaining equal, the apparent gravitational force in the slow condition of the present study is only about $11 \%$ of that seen normally. These slow walks, then, seem more appropriate to the moon, which has a gravity of $17 \%$ of earth's. Perhaps because there have been no American women astronauts to land on the moon (or few televised instant replays for women's sports), we have no experience at seeing differences between men and women moving under low-gravity-like conditions. If this analysis is correct, gender recognition would seem to require an accurate representation of the pendular movements of gait as we see them everyday.

Experiments 1 and 2 examined the importance of temporal parameters to processes of gender recognition. The next three experiments explore the contribution of two spatial parameters.

\section{EXPERIMENT 3 SPATIALLY DISCRETE VS. DIFFUSED REPRESENTATION OF JOINTS}

There are a number of ways to study the role of structural information in perception. One of these is to degrade the representation and to observe what decrement, if any, this degradation produces in recognition. Kozlowski and Cutting (1977, Experiment 5), for example, systematically removed pointsource information from gait displays. In all cases, however, recognition performance remained sig- 
nificantly above chance. Other methods of degradation have been developed in other domains. For example, in the study of face recognition, Harmon (1973) has used computer quantizing and filtering. High spatial frequencies are removed by parsing the photograph into individual points of brightness, averaging across a selected group of points, and then assigning a uniform brightness value to the specified area. The effect of this technique is to remove sharp edges and, hence, to blur the picture. Nevertheless, with an averaging window as large as $20 \%$ of portrait width, Harmon reported that recognition was still good. Thus, a large degree of systematic blurring does not markedly reduce facial recognition, and the conclusion to be gained is that the critical information in faces is holistic (low spatial frequency) in nature.

It may be that gender recognition in gait is relatively unaffected by blurring as well, and that a blurred version of the point-light array could provide sufficient representation of structural information. This proposition appears consistent with the report of Kozlowski and Cutting (1977, Experiment 5), who conclude that no single joint cue is necessary for gait recognition and that critical information seems to be distributed throughout the display.

\section{Method}

Four males and 11 females were paid $\$ 1$ apiece to view two test sequences. Following eight practice trials, the first sequence consisted of 28 standard trials like those used in Experiments 1 and 2. The second test consisted of a random sequence of 42 trials (three tokens per walker) in which the stimulus image was defocused. To produce the second tape, we focused the camera on the source monitor at a distance of approximately $2 \mathrm{~m}$. Then the aperture of the camera lens was opened to its widest (f/18.2), increasing light input to a maximum while narrowing the depth of field to a minimum. This resulted in diffusing the stimulus image so that all joint information was retained, but point lights were no longer separable into specific joint cues. Image contrast was adjusted so that it remained high. The total effect was not unlike that of Harmon's; the lights melted together, with each enlarged about five diameters, so that the effective blur, increased by some spatial summation, was about $20 \%$ of the monitor display. A static representation of the resulting stimuli can be seen in the lower portion of Figure 1. The upper portion of that figure shows the same walker without image diffusion.

\section{Results and Discussion}

Identifiability of standard stimuli was comparable to previous studies. Overall, viewers were able to judge gender of the walkers on $64 \%$ of all trials, $\mathrm{t}(14)=4.02, \mathrm{p}<.01$. Mean confidence scores for correct and incorrect trials were 2.2 and 1.9 , respectively, $\mathrm{t}(14)=2.63, \mathrm{p}<.02$. On the other hand, identifiability of the diffused stimuli did not differ from chance. On only $51 \%$ of all trials were walkers accurately identified, $\mathrm{t}(14)=.88$, and there was no difference in confidence scores for correct and incorrect trials, with a mean of 1.4 for each. A comparison of performance under standard and diffused conditions indicates a marked difference in viewers' ability to determine gender, $t(14)=3.8, p<.01$. Again, confidence scores reflect performance levels in the two conditions, $t(14)=6.06, p<.001$. Thus, although observers do not depend on particular joints for accurate judgment of gender, some aspects of joint cues must be a necessary source of information. When discrete joint cues are removed, leaving only the low-frequency "smooth, undulating flow of walking" (Napier, 1967, p. 57), the relevant perceptual information cannot be abstracted.

A second spatial attribute is the focus of the final two studies.

\section{EXPERIMENT 4 UPRIGHT VS. INVERTED DISPLAYS}

Experiment 2 varied presentation rate and analysis of those results implicated gravity in gender recognition. A second way to alter representation of gravitational forces is to invert the gait displays. This experiment was designed to analyze the importance of orientation by an up-down reversal of the stimuli. Inversion is known to yield odd effects in gait (see Krüger, cited in Kohler, 1964, p. 95).

\section{Method}

A test sequence of 64 trials -8 practice followed by a random ordering of four tokens per walker-was presented right-side up and upside down. An inversion of the stimulus image was produced simply by turning over the 9 -in. monitor used in all experimental sessions. Unlike the previous studies in this series, two groups of viewers participated in this study: Eight males and 7

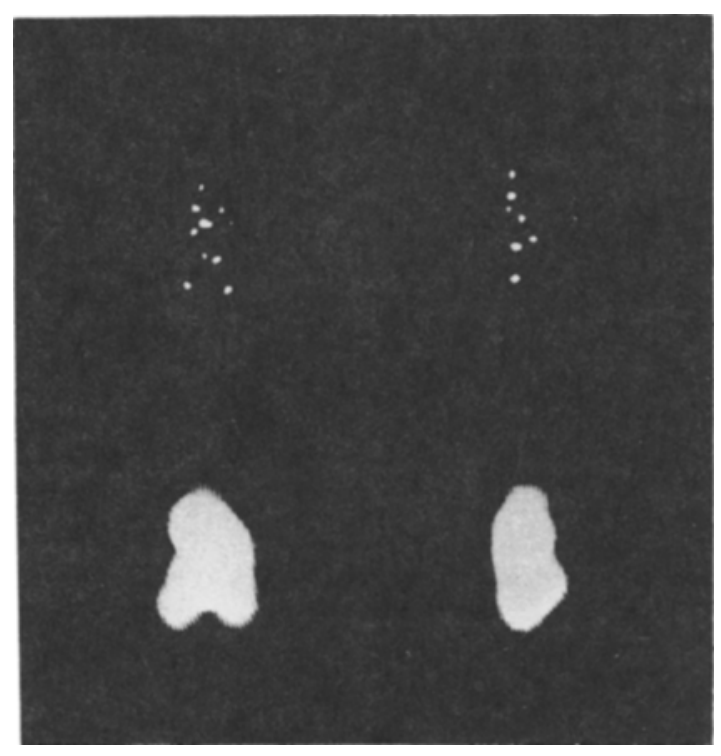

Figure 1. Static representations of a male walker shown in his most outstretched and most aligned positions within the step cycle, in both standard point-light and diffused conditions. 
females observed the standard stimuli, and 4 males and 11 females viewed the inverted stimuli. Each was paid \$1.

\section{Results and Discussion}

As expected, performance under the standard display condition was similar to that reported previously. Overall, correct identification was $65 \%$, $\mathfrak{t}(14)=4.97, \mathrm{p}<.001$. Mean confidence score for correct trials was 2.3 and for incorrect trials only $2.0, t(14)=3.78, p<.01$. Results for the inverted displays, however, were very surprising. Inversion not only impeded recognition but reduced performance to a level significantly below chance. Across all trials, the walkers were identified correctly only $39 \%$ of the time, $\mathrm{t}(14)=-5.0, \mathrm{p}<.001$. Disregarding the direction of this result, its deviation from $50 \%$ is not significantly different from the upright condition. Mean confidence score for correct responses was 1.3, whereas that for incorrect responses was 1.6. That is, viewers were significantly more confident when responding incorrectly, $\mathrm{t}(14)=-3.44, \mathrm{p}<.01$. Unlike the below-chance results of Experiment 1, these were systematic; identification of 12 of the 14 walkers reversed polarity, $z=2.41, p<.02$. That is, walkers identified as males rightside up were identified as females upside down, and rightside-up females were upside-down males. Overall, this result seemed so implausible that we felt that we must try to replicate it.

\section{EXPERIMENT 5 INVERTED DISPLAYS AGAIN}

\section{Method}

Seven males and eight females were paid $\$ 1$ apiece to view a test sequence of half the length of that used in Experiment 4. They viewed only the inverted displays, and they did not indicate confidence in their responses. Otherwise, procedures were identical to those used previously.

\section{Results}

Overall, viewers were $43 \%$ correct in their responses, $t(14)=-2.11, p<.04$, one-tailed. This result is significantly less than chance, like that of Experiment 4, but shows some regression towards the mean. This tendency, however, is attributable largely to the responses of one individual, who was $75 \%$ correct in her judgments, or 2.58 standard deviations above the mean. Apparently she had participated in at least one too many social psychology experiments. Upon later questioning, she volunteered that she had assumed we used male walkers to impersonate females, and vice versa. She seems to have adjusted her responses accordingly. If her data are omitted from statistical consideration, overall performance reduces to $41 \%, t(13)=-3.86, p<.002$.

When a comparison of viewer performance on each of the 14 walkers is made between this study and that for the inverted condition in Experiment 4, a striking correlation is found, $r=.79, t(12)=$ $4.96, p<.001$. Thus, the reversal of gender identification in inverted conditions appears to be no fluke, and must be accounted for.

\section{GENERAL DISCUSSION}

\section{Overview}

The results of Experiment 1 showed that the temporal threshold for gender recognition of a walker is greater than that required for simple perception of a person in motion. With a 4.4-sec dynamic display, performance was $66 \%$ whereas at all briefer exposures it was very near to chance. This threshold appears to lie within a range of 1.6 to $2.7 \mathrm{sec}$, or roughly 2 step cycles. The results of Experiment 2 corroborated those findings reported by Kozlowski and Cutting (1977, Experiment 3) in that viewers cannot identify a walker's gender when the natural cadence of individual gait is violated. We suspect that alteration of presentation rate changes apparent gravitational force and obscures the relationship between output and conservation of energy in natural walk. On the basis of the results reported in Experiment 3 , it seems reasonable to conclude that gender recognition depends in some way on specific joint relations in the dynamic stimulus. Enlarging point lights so that they lose their separate integrity appears to conceal sources of sex-related information. The results of Experiments 4 and 5 support the proposition, raised in Experiment 2, that gender recognition includes references to gravitational orientation. As in all standard conditions, performance hovered around $65 \%$. Most surprisingly, however, inverted displays systematically reversed the identifiability of nearly all walkers. Conjecture as to the cause of this reversal would, at this point, be premature. The discussion to follow makes an attempt to account for all results.

\section{Some Other Considerations}

Two facts lead us toward an explanation of how gender recognition is done without familiarity cues. First, Kozlowski and Cutting (1977, Experiment 5) reported that recognition is best when joints from both upper and lower portions of the body are represented in the dynamic point-light display, but that upper-body information alone yielded significantly higher performance than lower-body information. Second, viewers who participated in Experiment 1 of this series were asked a series of questions concerning gait. Among them was "Which parts of the body, shoulders or hips, do you think are the most prominent in the walks of males and females?' Of that group, $76 \%$ reported shoulder movement accentuated for males and $100 \%$ hip movement for fe- 
males. Perhaps structural information about the shoulders and hips contributes to gender recognition. On the basis of this possibility, it seemed appropriate to measure the widths of the shoulders and hips of each of the 14 walkers in this study. Indeed, these measurements serve to clarify the influence of relative joint information on gender recognition.

\section{Structural Differences Between Males and Females}

Tape measurements of each walker were taken on two separate occasions to maximize reliability. They included the distance across the front of the shoulders (marked on each side by the acromion, or most laterally protruding point of the scapula) and the distance across the front of the pelvis (marked on each side by the most laterally protruding point of the iliac crest). These pelvic measurements are commonly used to describe the hips, but technically the hip joint is defined by the intersection of the femur with the pelvis, about $10 \mathrm{~cm}$ below this portion of the ilium. This higher point was used here simply because it was easier to measure and closer to the point at which the reflectant tape was mounted.

To provide a consistent measure of gender identifiability, the percentage of trials for which each of the 14 walkers was identified as male was computed and compared for standard conditions in the first four experiments. The coefficient of concordance, $\mathrm{W}=.72, \chi^{2}(13)=37.5, \mathrm{p}<.001$, indicates the high reliability of these findings. These percentages were then weighted according to the number of viewers in each experiment and averaged, yielding a mean "percent maleness" score for each of the 14 walkers." Measurements and percent maleness scores are shown in Table 1.

When male and female measurements are compared, it can be seen that males' shoulders are wider than females' by $3.6 \mathrm{~cm}, \mathrm{t}(12)=5.63, \mathrm{p}<.001$, and that females' hips are slightly but not significantly wider than males', $\mathrm{t}(12)=-.79$, n.s. The difference between shoulder and hip widths, shown in Column 3, is quite striking across groups, $\mathrm{t}(12)=4.29, \mathrm{p}<$ .001 , and can be used as an index of torso structure. ${ }^{5}$ When this structural information is correlated with percent identification as male (the last column of Table 1), the correspondence between the two measures is striking, $r=.87, t(12)=6.15, p<.001$. It appears, then, that gender recognition may depend to a large degree on a biomechanical difference in males and females, the locus of which appears to be in the structural relations. of the shoulders and hips.

This possibility raises an interesting paradox. All walkers in these experiments and those reported by Kozlowski and Cutting were viewed from the side. Thus, one might think that structural information about shoulders and hips would not be visible to the viewer, since only the right hip and right shoulder were seen. If structural differences between males and females contribute to identifiability, then they must be manifested in an alternate form.

Carlsöö (1972), among others, describes human gait as a cycle of alternative movements. Arms move in opposition to legs, and the torso, acting very much like a flat spring, bends in one direction at the top (the shoulders) to follow the arms and in the opposite direction at the bottom (the hips) to follow the legs. This torsion is perfectly correlated with other movements within the gait cycle. Since males' shoulders are wider than females', the reflectant patches on male shoulders might describe a wider arc than those for females. Correspondingly, since female hips are slightly broader, these subtle differences might be perceived by viewers and used as primary sources for decision making. Such torsion at the shoulders and hips would be incompletely revealed in the brief exposures of Experiment 1, displayed in an unnatural cadence in the slow condition of Experiment 2, and hidden in the diffused condition of Experiment 3.

Given that such torsion might explain the results of the first three Experiments, it is tempting to try to account for the results of Experiments 4 and 5 in a similar way: (1) If, because of the structural differences shown in Table 1 , males move their shoulders more than females and females move their hips slightly more than males, and (2) if, upon inversion, the movement of a walker's shoulders are seen as if they were hips and the hips are seen as if they were

Table 1

Measurements (in Centimeters) of the Walkers as Compared Against Their Identifiability

\begin{tabular}{|c|c|c|c|c|}
\hline Walkers & Shoulders & Hips & $\begin{array}{c}\text { Shoulders- } \\
\text { Hips }\end{array}$ & $\begin{array}{c}\text { Percent } \\
\text { Maleness }\end{array}$ \\
\hline \multicolumn{5}{|c|}{ Females } \\
\hline $\begin{array}{l}\text { 1. T.R. } \\
\text { 2. I.B. } \\
\text { 3. D.D. } \\
\text { 4. A.L. } \\
\text { 5. J.S. } \\
\text { 6. E.L. } \\
\text { 7. C.P. }\end{array}$ & $\begin{array}{l}38.0 \\
38.5 \\
38.5 \\
37.0 \\
38.5 \\
37.5 \\
39.5\end{array}$ & $\begin{array}{l}39.5 \\
42.0 \\
35.0 \\
36.0^{*} \\
40.0 \\
39.0 \\
40.5\end{array}$ & $\begin{array}{r}-1.5 \\
-3.5 \\
3.5 \\
1.0 \\
-1.5 \\
-1.5 \\
-1.0\end{array}$ & $\begin{array}{l}34.6 \\
20.0 \\
36.6 \\
45.7 \\
47.4 \\
25.5 \\
35.5\end{array}$ \\
\hline $\begin{array}{l}\text { Mean } \\
\text { SD }\end{array}$ & $\begin{array}{r}38.2 \\
.8\end{array}$ & $\begin{array}{r}38.8 \\
2.4\end{array}$ & -.6 & $\begin{array}{r}35.0 \\
9.9\end{array}$ \\
\hline \multicolumn{5}{|c|}{ Males } \\
\hline $\begin{array}{l}\text { 8. R.E. } \\
\text { 9. R.K. } \\
\text { 10. E.A. } \\
\text { 11. D.W. } \\
\text { 12. R.R. } \\
\text { 13. A.M. } \\
\text { 14. R.W. }\end{array}$ & $\begin{array}{l}40.5 \\
40.0 \\
41.0 \\
43.0 \\
43.0 \\
44.0 \\
41.0\end{array}$ & $\begin{array}{l}38.0 \\
38.0 \\
36.5 \\
38.5 \\
40.0 \\
38.0 \\
38.0\end{array}$ & $\begin{array}{l}2.5 \\
2.0 \\
4.5 \\
4.5 \\
3.0 \\
6.0 \\
3.0\end{array}$ & $\begin{array}{l}57.0 \\
54.0 \\
68.9 \\
67.8 \\
73.8 \\
75.5 \\
59.5\end{array}$ \\
\hline $\begin{array}{l}\text { Mean } \\
\text { SD }\end{array}$ & $\begin{array}{r}41.8 \\
1.5\end{array}$ & $\begin{array}{r}38.1 \\
1.0\end{array}$ & $\begin{array}{l}3.6 \\
1.4\end{array}$ & $\begin{array}{r}65.2 \\
8.4\end{array}$ \\
\hline
\end{tabular}

*Walker 4 was not present for the second group of hip measurements so that hers is estimated on the basis of the average difference in first and second measurements for female walkers. 
shoulders, then viewer's responses would reverse with respect to the true gender of the walker but the magnitude of the result (as compared against chance) would remain the same. Unfortunately, the index of torso structure is not significantly correlated with the degree to which each walker is identified as male when presented upside down, $r=-.19$, n.s. Thus, some other attribute must account for these data. We suspect that since inverted walkers are seldom experienced and, as Kohler (1964) noted, inverted human movement has an unnatural and arhythmic appearance to it, viewers in these conditions are forced to adopt a mode other than that of perceiving torsion. What the basis for that mode is, we have been unable to discover. ${ }^{6}$

\section{CONCLUSION}

We believe that gender recognition of a walker from dynamic point-light displays such as those used in the standard conditions of these experiments is accomplished, in part, with reference to movements of the shoulder and hip. Support for this notion stems from four facts presented here and elsewhere: (1) Viewer performance is best when lights are attached to both shoulder and hip, (2) males' shoulders in our sample are significantly broader than females' and females' hips are slightly broader than males', (3) since the torso twists during natural gait, these differences would become visible to the viewer, and (4) viewers believe that males move their shoulders more than females and that females move their hips more than males. Following this logic, Cutting, Proffitt, and Kozlowski (1978) provide a series of specifications as to how invariant information might be represented in point-light displays.

\section{REFERENCES}

BecketT, R., \& Chang, K. An evaluation of the kinematics of gait by minimum energy. In D. Bootzin \& H. C. Muffley (Eds.), Biomechanics. New York: Plenum, 1969.

BERNSTEIN, N. The co-ordination and regulation of movements. London: Pergamon, 1967.

Birdwhistell, R. L. Kinesics and context. Philadelphia: University of Pennsylvania Press, 1970.

Bornstein, M. H., \& Bornstein, H. G. The pace of life. Nature, 1976, 259, 557-559.

Carlsöö, S. How man moves. London: Heinemann, 1972.

Cutring, J. E., \& Kozlowski, L. T. Recognizing friends by their walk: Gait perception without familiarity cues. Bulletin of the Psychonomic Society, 1977, 9, 353-356.

Cutting, J. E., Proffitt, D. R., \& Kozlowski, L. T. A biomechanical invariant for gait perception. Journal of Experimental Psychology: Human Perception and Performance, 1978,4 , in press.

FARBer, J. The student as nigger. New York: Pocket Books, 1969.

Gray, H. Anatomy, descriptive and surgical. New York: 1977 (15th ed. originally published 1901).

Grillner, S. Locomotion in vertebrates: Central mechanisms and reflex action. Physiological Review, 1975, 55, 247-304.
Harmon, L. The recognition of faces. Scientific American, 1973,229 (5), 70-84.

Johansson, G. Visual perception of biological motion and a model for its analysis. Perception \& Psychophysics, 1973, 14, 201-211.

Johansson, G. Visual motion perception. Scientific American, 1975,232 (6), 76-89.

Johansson, G. Spatio-temporal differentiation and integration in visual motion perception. Psychological Research, 1976, 38, 379-393.

Jones, M. R. Time, our lost dimension: Toward a new theory of perception, attention, and memory. Psychological Review, $1976,83,323-355$.

KoHLER, I. The formation and transformation of the perceptual world. Psychological Issues, 1964, 3(4), 1-173.

Kozlowski, L. T., \& Cutring, J. E. Recognizing the sex of a walker from a dynamic point-light display. Perception \& Psychophysics, 1977, 21, 575-580.

NAPIER, J. The antiquity of human walking. Scientific American, 1967, 216(4), 56-66.

STEINDLER, A. Mechanisms of normal and pathological locomotion in man. Springfield, Ill: Thomas, 1935.

\section{NOTES}

1. Due to separate recording sessions for male and female walkers, some systematic variation across sexes occurred. However, apparent size of the males was slightly smaller (by $8 \%$ ) than that of the females, opposite from normal variation of the two samples. Thus, in some sense, this variation was loaded against us in obtaining significant results. Because overall results here are so comparable to our previous results (Kozlowski \& Cutting, 1977), where there was no such variation, we believe that this factor contributed little, if anything, to the patterns of results reported here.

2. All t tests reported here are two-tailed, unless otherwise indicated.

3. Since the legs and arms are compound pendula, their length can vary. For example, when one runs, he or she usually shortens the arm pendula by bending the elbows, and shortens a leg pendulum in the swing phase by bending the knee. Both gestures shorten the distance between pivot and center of mass, allowing for more rapid swinging.

4. Of course, percent femaleness could just as well have been used.

5. One might argue that, since it is only the shoulder widths that differ significantly for males and females in our sample, the index for torso structure need not concern hip width. Indeed, when shoulder width is correlated with percent maleness, a strong relation is seen, $r=.84$. We include the hips here in part because we believe that our sample of men and women may not accurately represent relative hip widths. For example, Gray's Anatomy (1901/1977, pp. 178-181) states that women's pelvises are usually wider than males'. Moreover, inclusion of the hips in this index may indirectly allow inclusion of a gender presentation difference noted by Birdwhistell (1970, p. 44). He notes that the American male tends to carry his pelvis rotated slightly backward and that the American female rotates hers slightly forward. It seems likely that this factor could affect hip movement revealed in natural gait as well as in the dynamic point-light display.

6. It is possible that differences in apparent size of males and females (see Footnote 1), with males slightly smaller than females, contributed to the inverted results. Pooling the results of Experiments 4 and 5 and calculating "inverted percent maleness" for each walker yields an index that is marginally correlated with visual angle of each walker/stimulus, $r=.44, t(12)=1.69$, $\mathrm{p}<.10$.

(Received for publication June 29, 1977; revision accepted November $21,1977$. ) 